\title{
Research on Vehicle High Speed Motion Stability and Simulation Based on Computer Aided Design
}

\author{
Zhensheng Zhang ${ }^{1}$, Hailing Han ${ }^{2}$, Rui Li ${ }^{3}$, Xingyuan Zhang ${ }^{1}$ \\ ${ }^{1}$ Chongqing College of Electronic Engineering, Chongqing, China. \\ ${ }^{2}$ Liaoning Provincial College of Communications, Liaoning, China. \\ ${ }^{3}$ Chongqing Vocational Institute of Engineering, Chongqing, China.
}

Keyword: Computer Aided Design, High Speed Motion Stability, Vehicle

\begin{abstract}
In this paper, through the research on high speed moving vehicle aerodynamic stability by using the method of computer aided design, the result of the research has showed that there is a close relationship between the automobile body surface pressure distribution and automobile shape, which can have a direct impact on the vehicle's aerodynamic performance. In front of the hood and the front cover, it is the high-pressure area, the pressure is higher than the pressure of the end of the vehicle, which is the main reason for the formation of aerodynamic resistance, moreover, it has made detailed analysis on the impact of the automobile air pressure distribution on the aerodynamic characteristics.
\end{abstract}

\section{Introduction}

When vehicle is running at high speed, it often can be encountered with the problem of "unstable state",this high-speed aerodynamic instability phenomena, namely, the driver feel that the driving vehicle has lost road feeling, meanwhile the steering system of the vehicle has slow response, then the driver can completely lose the control of the vehicle[1]. The direct reason that caused the "unstable state" is the aerodynamic lifting power can overcome the weight of vehicle, perpendicularly holding it to the ground, making the adhesion power between wheels and the ground poor[2-3]. Therefore, during the process of aerodynamic design of high speed vehicle, aerodynamic lift coefficient is very important, which has a great influence on the stability and safety of vehicle. The vehicle with higher aerodynamic lift coefficient will cause the wheel to lose a part of the grip power when it is at high speed, moreover, it may even lead to a serious loss of control which can cause traffic accidents. However, good aerodynamic design, which may even make the aerodynamic lift coefficient of the vehicle be negative, the aerodynamic negative lift power can be increased with the increase of the vehicle speed, which can ensure the vehicle move at high speed.

This paper aims to explore the causes of aerodynamic lift, analyzing vehicle flow field distribution, making deep study on the influencing factors of aerodynamic lift, so as to design the corresponding aerodynamic equipment and improve the aerodynamic lift, which can increase the negative lift of the vehicle when it is driving at high speed, it can have important engineering significance on improving the stability of vehicle at high speed. With the improvement of modern computer technology and the continuous development of the theory of turbulence, it makes Computational Fluid Dynamics (CFD) be widely used during the development of automotive aerodynamics. This paper is based on CFD simulation technology, making analysis on the impact mechanism of aerodynamic lift of of vehicle, so as to make deep research on the improved design of the additional device to the aerodynamic lift. 


\section{Materials and Methods}

Optimization Method Based on Approximate Model

Because the geometric parameters of the vehicle surface is complex, the relationship between the parameters and the aerodynamic characteristics of the vehicle can not be determined. The traditional aerodynamic optimization method generally adopts the method of design----evaluation-----design. This kind of optimization method is mainly depended on the experience of the designers, moreover, the efficiency is low, which can cost a lot of development time. With the development of computer technology, the optimization design of numerical algorithm is applied to the development of modern products. Through the numerical calculation method, the optimization design can automatically find out the best solution to determine the boundary conditions, the optimization process can be carried on automatically without the required manual intervention. Thus, it can shorten the cycle of designing the products and avoid the occurrence of human error.

With the development of CAD and CFD technology, the simulation of the automobile aerodynamic computer is more and more close to the actual situation, and the calculation of aerodynamic force is more accurate. Therefore, one method that can realize the optimization method of multidisciplinary coupling has been emerged ----- aerodynamic optimization method based on approximate model. Approximate method is using the known sample points of information to predict the response of the unknown point value, its essence is to make the discrete quasi be the mathematical approximation model, which can effectively make the non-smooth mapping between parameters variable and the objective function become the approximated smooth mapping. Through this approximate model, it can get the best solution by using the method of having numerical calculation. The basic steps can be summarized as follows:

(1) Fixing the determination of the shape of vehicle as well as the flow region, so as to form the calculation area of CAD model.

(2) Dividing the calculation domain space into polyhedron mesh.

(3) Determining the calculation equation and calculation model.

(4) Determining the boundary conditions, including the entrance and exit of the boundary conditions, as well as wall boundary conditions. Boundary conditions in mathematics should meet the feature of adaptability, in the physical sense, it should have practical significance.

(5) Setting the specific solution process and the accuracy of some control parameters and conditions, having numerical simulation to the flow field.

(6) Reading the files of calculation results, so as to get the aerodynamic drag coefficient and lift coefficient. Then the pressure chart and velocity vector chart as well as the velocity flow chart can be analyzed.

\section{The Establishment of Experimental Model}

(1) Introducing CAD Model of Vehicle

In order to make better study on the the function of diffuser, in this paper, it selects the closed vehicle chassis, a certain brand of sportscar which has little gap between the ground and the model, which can be shown in Fig.1, hereinafter it refers to as A model, which can be as the researching object. The length and breadth and height is $4206 \times 1775 \times 1452(\mathrm{~mm})$, the minimum gap between ground is $108(\mathrm{~mm})$. 


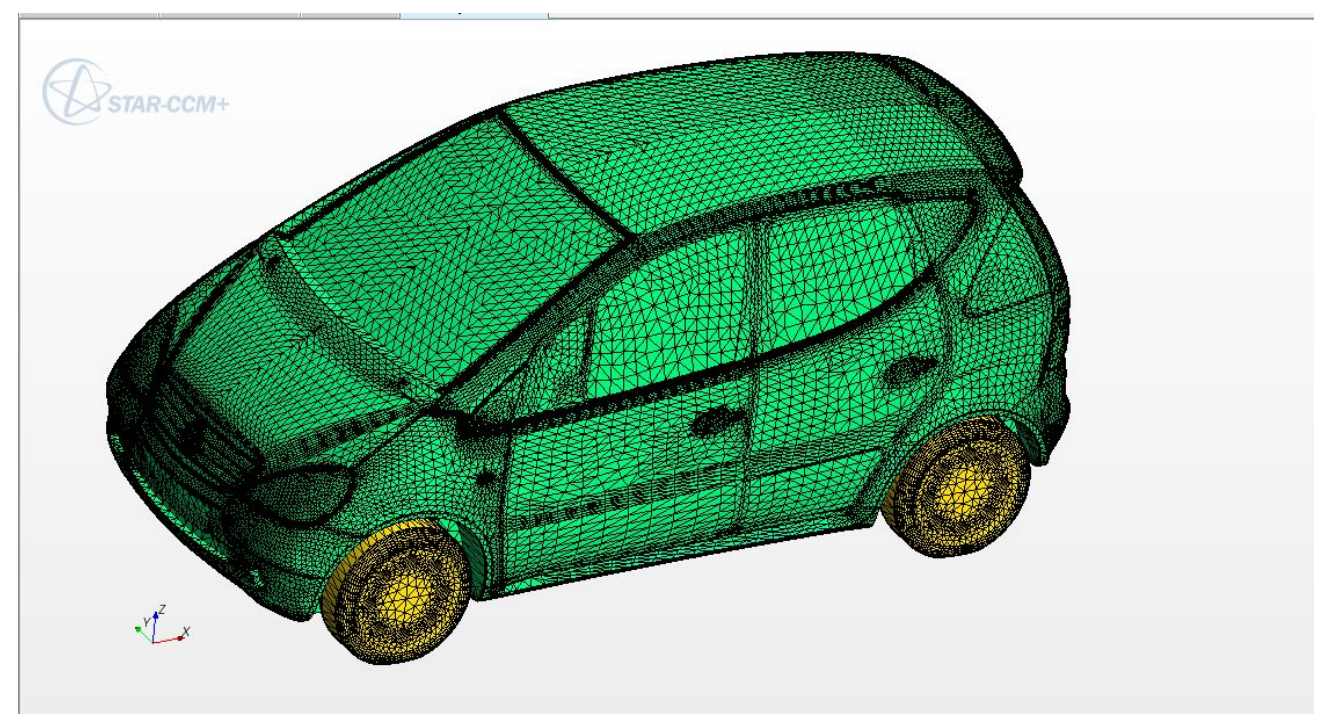

Fig. 1 CAD Model of Vehicle

(2) Establishing the flow field

Due to the symmetry of this model, the symmetric mode can be used to calculate. See Fig. 2.

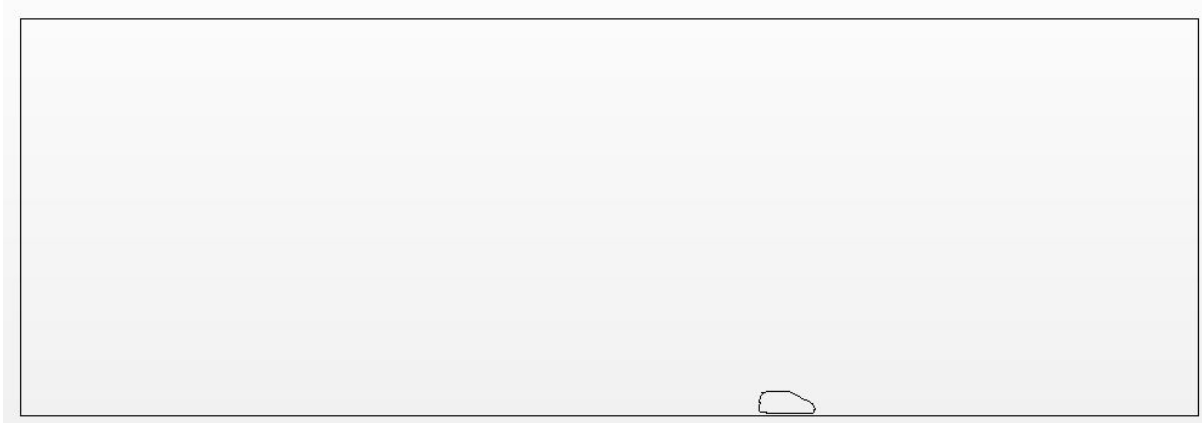

Fig. 2 Schematic Diagram of Flow Field

(3) Introducing the model into the flow field, then the model of vehicle and the flow field can be divided by means of grid, the result can be shown in Fig. 3 and Fig. 4.

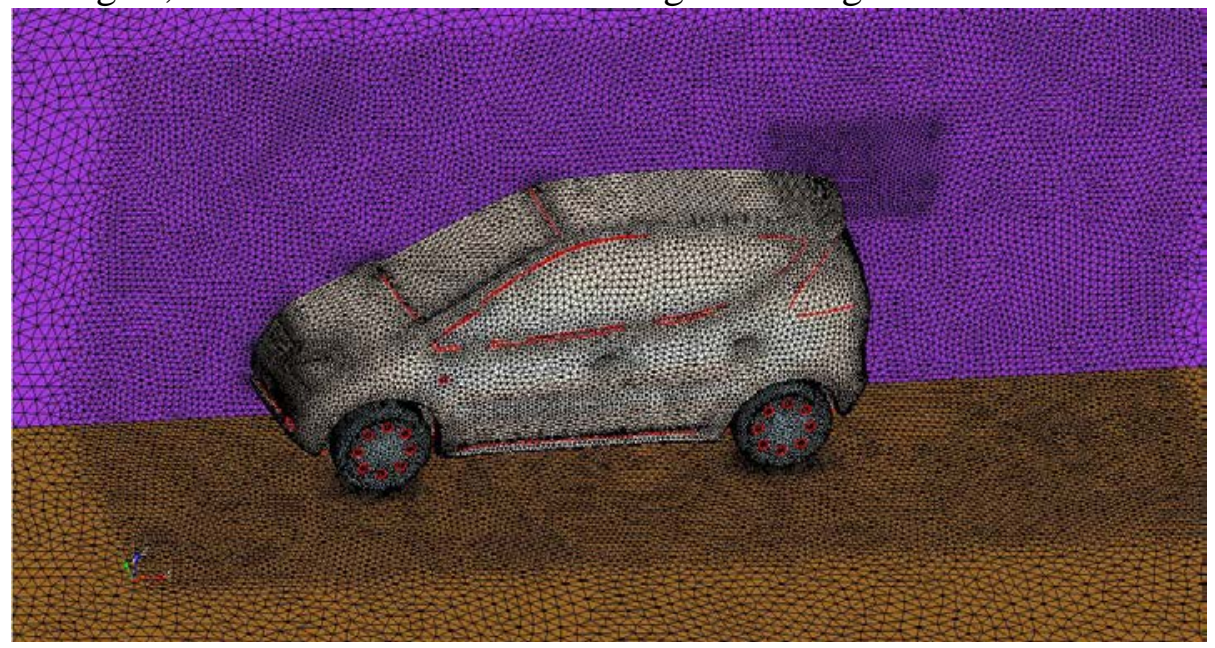

Fig. 3 The Division of Surface Mesh 


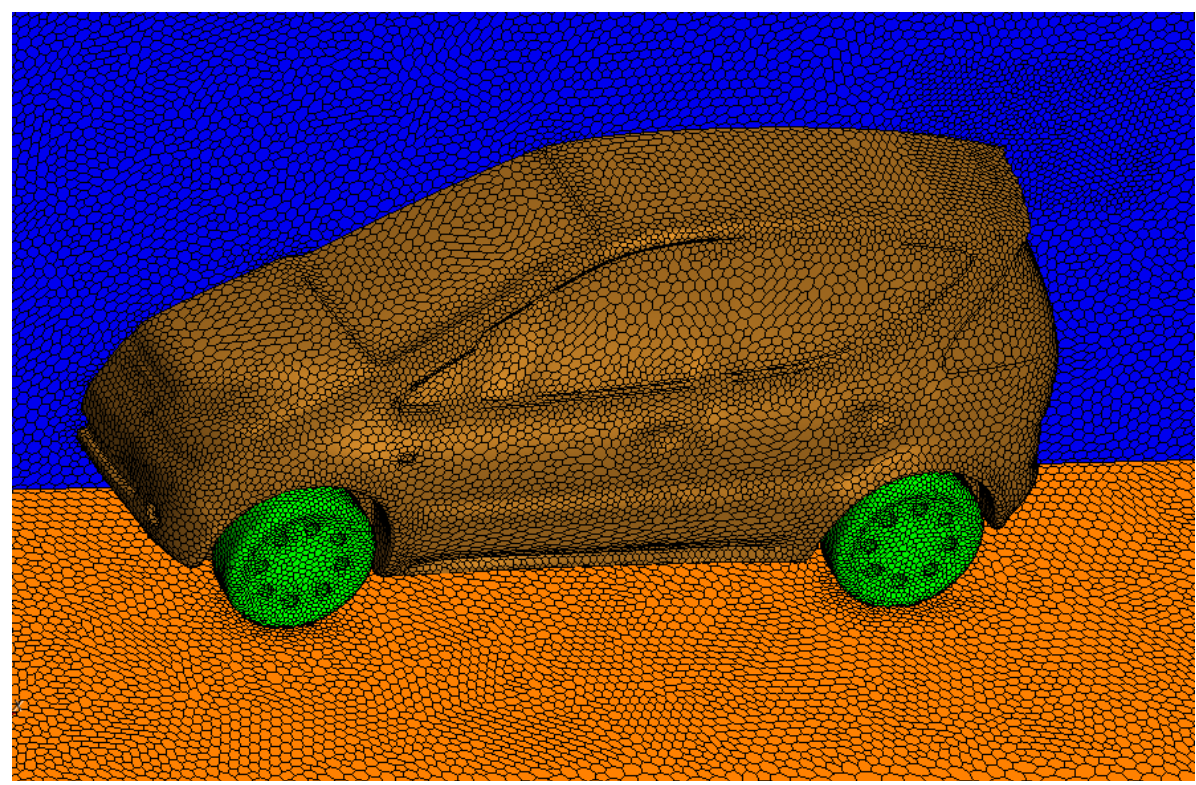

Fig. 4 Polyhedral Mesh

\section{Numerical Simulation Results and Analysis of the External Flow Field of Vehicle}

External boundary conditions for the numerical simulation of the external flow field of the vehicle can be shown in Table 1.

Table 1 External Boundary Conditions for the Numerical Simulation of the External Flow Field of the Vehicle

\begin{tabular}{|c|l|}
\hline Boundary of calculation domain & Set Value \\
\hline Entry of calculation domain & $\begin{array}{l}\text { v= speed of operating condition } \\
\text { Turbulence intensity } I=0.03 \% \\
\text { Characteristic scale: } 1=0.01 \mathrm{~m}\end{array}$ \\
\hline Exit of calculation domain & Pressure outlet \\
\hline Ground & No slipping wall \\
\hline $\begin{array}{l}\text { The top and upper side of calculation } \\
\text { domain }\end{array}$ & Slipping wall \\
\hline The surface of vehicle & Wall without slipping \\
\hline Fluid & $\begin{array}{l}\text { Air: density } \rho=1.18415 \mathrm{~kg} / \mathrm{m}^{3} \\
\text { Viscosity } \mu=1.85508 \mathrm{E}-5 \mathrm{~Pa}-\mathrm{S}\end{array}$ \\
\hline
\end{tabular}

Having simulation on the vehicle at the speed of of $90 \mathrm{KM} / \mathrm{H} 、 120 \mathrm{KM} / \mathrm{H} 、 150 \mathrm{KM} / \mathrm{H}$ 、 $180 \mathrm{KM} / \mathrm{H}$, then it can get the pressure cloud chart, velocity vector chart and streamline chart, which also can be analyzed.

Aerodynamic drag coefficient and lift coefficient at different velocities.

Through simulation, it can get aerodynamic drag $D$ and aerodynamic dragcoefficient $C_{D}$ of vehicle at the speed of 90KM/H、120KM/H、150KM/H、180KM/H see Table 2. 
Table 2 Aerodynamic drag $D$ and aerodynamic drag coefficient $C_{D}$ of vehicle at the speed of $90 \mathrm{KM} / \mathrm{H} 、 120 \mathrm{KM} / \mathrm{H} 、 150 \mathrm{KM} / \mathrm{H} 、 180 \mathrm{KM} / \mathrm{H}$

\begin{tabular}{|c|c|c|}
\hline $\begin{array}{c}\text { Simulated vehicle speed } \\
(\mathrm{KM} / \mathrm{H})\end{array}$ & $\begin{array}{c}\text { Aerodynamic drag } \\
\text { coefficient }\end{array}$ & Lift coefficient \\
\hline 90 & 0.2952 & 0.0393 \\
\hline 120 & 0.2949 & 0.0388 \\
\hline 150 & 0.2937 & 0.0382 \\
\hline 180 & 0.2941 & 0.0389 \\
\hline mean value & 0.294475 & 0.038800 \\
\hline
\end{tabular}

Pressure Cloud Chart can be shown in Fig. 5

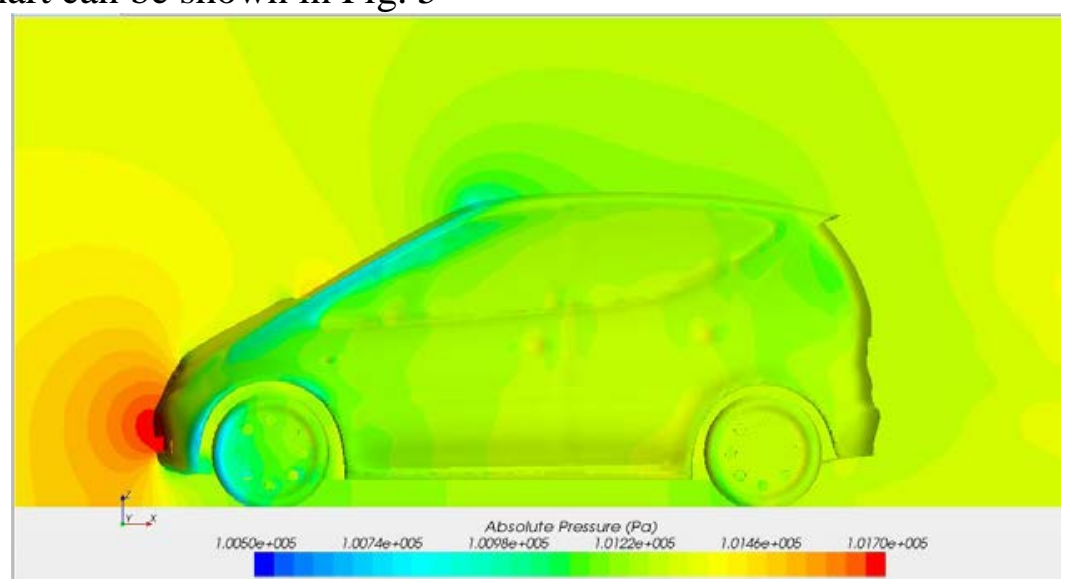

Fig.5 Pressure Cloud Chart of Vehicle at the Speed of 90KM/H

The surface pressure distribution of automobile body is closely related to the automobile shape, which can directly affect the aerodynamic performance of the vehicle. In the place of the front part, as well as the front hood is the high pressure area, the pressure is higher than the pressure of the rear part of the vehicle obviously,which is the main reason for the formation of aerodynamic resistance. The pressure from the upper part of the vehicle is less than the pressure of the bottom, thus, the pressure difference can form the upward lift force. The speed streamline chart and velocity vector chart of the vehicle can be shown in Fig. 6 and Fig.7.

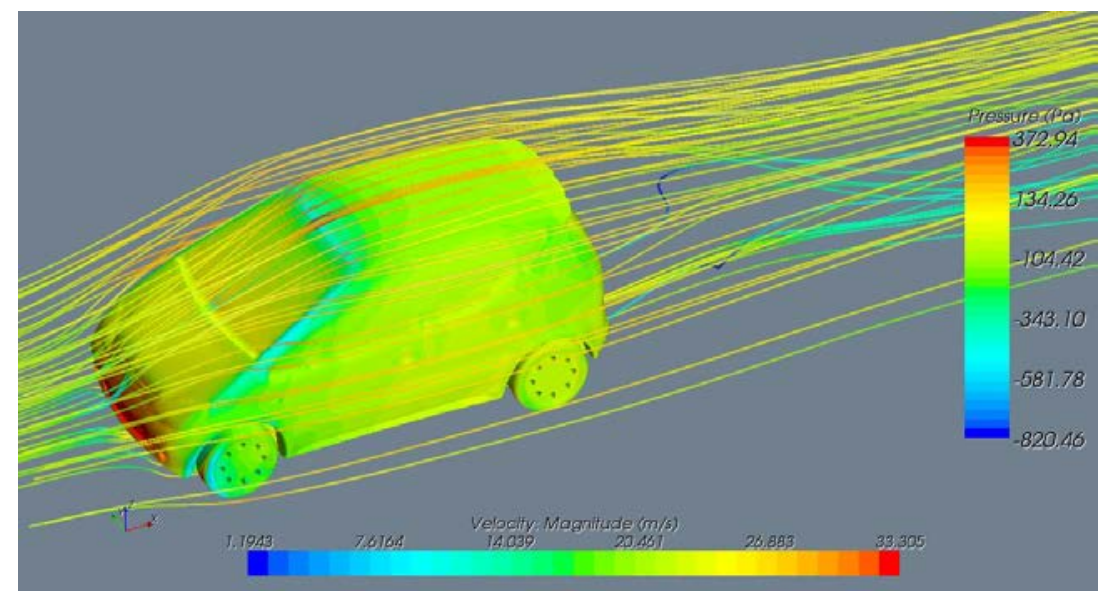

Fig.6 The Streamline Chart of Vehicle at the Speed of 90KM/H 


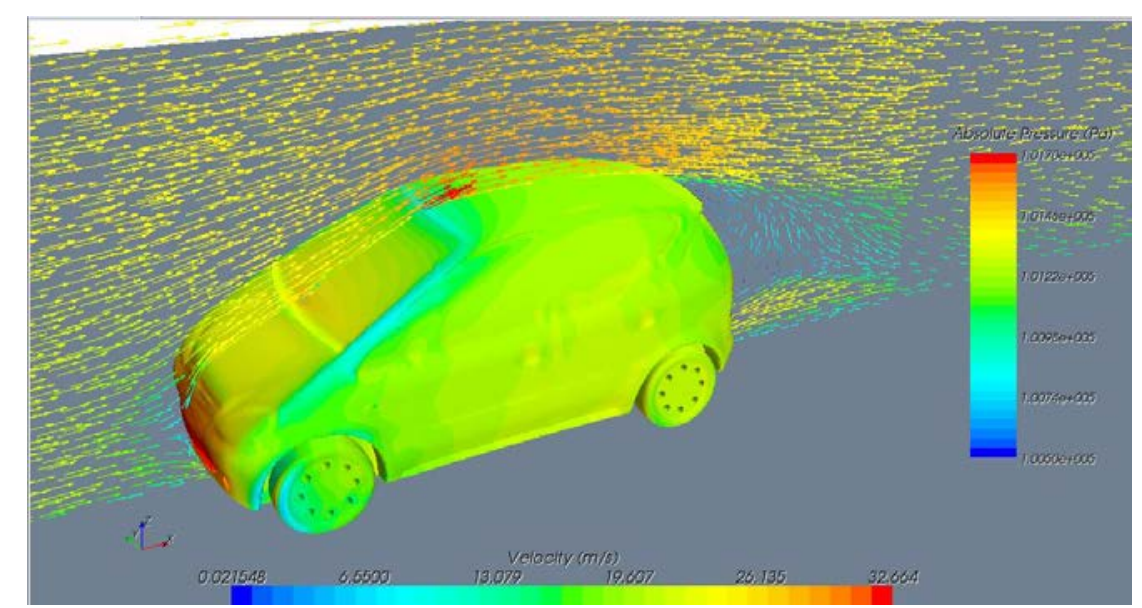

Fig. 7 The Velocity Vector Chart of Vehicle at the Speed of 90KM/H

The airflow from the front can contact with the front part of the vehicle, which can cause a certain air stagnation, the rate of the airflow can be decreased, beginning to flow and separate. Then the airflow of the front face can be combined with the downward airflow at the bottom of the vehicle, forming a vortex at the rear part of the vehicle; producing a smaller airflow separation zone, when the airflow of the front face is through the front of the hood and windshield glass with the front edge junction of the top roof, which can make the speed of airflow too high. The existence of the vortex from the rear part of vehicle can cause considerable energy loss.

\section{Conclusion}

This study has established automobile lift simulation model with the high speed movement, it takes the interfered flow of model vehicle as the calculating example to study the influence rules of "ground effect" on the aerodynamic lift,through CFD study of the simplified vehicle bluff body model, making a detailed analysis on the influence rules of the effect of the distribution of the automobile air pressure on the aerodynamic characteristics of vehicle. Therefore, it can play the role of having good theoretical foundation and engineering guidance for the improvement of the aerodynamic lift of the actual vehicle.

\section{Acknowledgements}

Chongqing Municipal Education Committee, Science and Technology Research Project, the contract number: KJ122205

\section{Reference}

[1] Isermann, R., J. Schaffnit, and S. Sinsel. "Hardware-in-the-loop simulation for the design and testing of engine-control systems." Control Engineering Practice 7.5 (1999): 643-653.

[2] Vogeltanz, Tomáš. "A Survey of Free Software for the Design, Analysis, Modelling, and Simulation of an Unmanned Aerial Vehicle." Archives of Computational Methods in Engineering (2015): 1-66.

[3] Su, Yi-peng. "Review of Craniofacial Plastic Correction Method Based on Computer Aided Technology." Journal of Applied Science and Engineering Innovation 2.11 (2015): 431-434. 